\title{
Rheumatic Fever and Systemic Lupus Erythematosus: A Rare Association
}

\author{
Jaouad Yousfi $^{1 *}$, Amal Elwakhomi ${ }^{1}$, Mouna Zahlane ${ }^{1}$, Lamiaa Essaadouni ${ }^{1}$

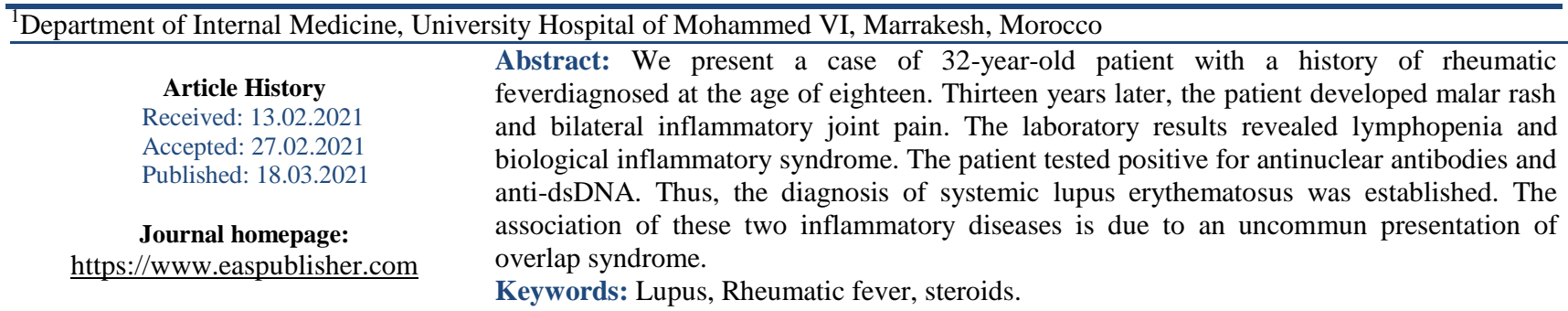

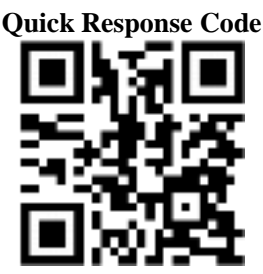

Copyright (C) 2021 The Author(s): This is an open-access article distributed under the terms of the Creative Commons Attribution 4.0 International License (CC BY-NC 4.0) which permits unrestricted use, distribution, and reproduction in any medium for non-commercial use provided the original author and source are credited.

\section{INTRODUCTION}

Rheumatic fever (RF) is an inflammatory systemic disease triggered by the group A-hemolytic Streptococcusthat occurs in both children and adults. It is characterized by rheumatic involvement and lifethreatening cardiac involvement. The association of RF and systemic lupus erythematosus (SLE) has been exceptionally reported in the literature. We report a case of SLE occurring 14 years after the onset of RF in a 32year-old patient.

\section{Case Presentation}

At the age of 18 , a patient presented with fever, inflammatory joint pain and mitral valve disease. The diagnosis of RF was made based on the Jones criteria, and she was treated with intramuscular benzathine penicillin G; 1.2 million I.U every 21 days.

Thirteen years later, the patient presented with bilateral inflammatory joint pain and photosensitivity. On physical examination, she was apyretic. She had a malar rash and stiff joints involving knees, elbows and wrists. No murmurs were heard on auscultation.

Biological assessment results showed a lymphopenia $\left(820 / \mathrm{mm}^{3}\right)$, a C-reactive protein (CRP) at $32 \mathrm{mg} / \mathrm{l}$, and an erythrocyte sedimentation rate of 52 $\mathrm{mm}$. Antinuclear autoantibodies were positive at 1/640 with homogeneous pattern. Anti-dsDNA were also positive at 120 (threshold of 30). The 24-hour urine protein was negative. No haematuria orleukocyturia were observed. Cardiac ultrasound confirmed mitral valve disease, without signs of endocarditis or pericarditis.

Low dose steroid therapy was started $(20 \mathrm{mg}$ of prednisone daily), in association with synthetic antimalarial drugs.Benzathine penicillin $G$ was maintained. The follow-up showed an improvement of the skin and joints features.

\section{DISCUSSION}

The association between SLE and RF suggests a physiopathological and clinical linkbetween these two inflammatory diseases.According to some authors, there is an overlap of humoral immunity against autoantigens such as human cardiac myosin [1].

Sera from SLE and poststreptococcal acute glomerulonephritis patients demonstrated idiotypic reactivity with anti-My1.A study showed that affinitypurified anti-myosin antibodies from SLE and RF sera also reacted strongly with anti-Myl, indicating that immunoglobulins produced in these diseases share idiotypic determinants. The data demonstrated an association of the Mylidiotype with poststreptococcal sequelae and SLE [2]. 
The review of the literature revealed two cases (a 15 year-old male and a 16 year-old female) with RF who subsequently developed SLE [3, 4].

In our case, the diagnosis of RF was certain, and the patient was treated by benzathine benzylpenicillin (penicillin G) 2.4 million units every 21 days. The possibility of SLE at this stage is unlikely, given the clinical presentation and recurrent tonsillitis episodes. The diagnosis of SLE was based on cutaneous manifestations (photosensitivity andmalar rash), joint pain, with negative anti-streptolysin $\mathrm{O}$ and positive antinuclear antibodies and anti-dsDNA. The association of SLE and RF poses a therapeutic challenge. Our patient should keep the antibiotherapy to avoid heart failure, as well as oral corticosteroid therapyand synthetic antimalarial drugs.

\section{CONCLUSION}

Our observation reports a rare association of SLE with RF. Further investigation could necessary to study this rare presentation of overlap syndrome.

\section{REFERENCES}

1. Blank, M., Krause, I., Magrini, L., Spina, G., Kalil, J., Jacobsen, S., ... \& Shoenfeld, Y. (2006). Overlapping humoral autoimmunity links rheumatic fever and the antiphospholipid syndrome. Rheumatology, 45(7), 833-841.

2. McCormack, J. M., Crossley, C. A., Ayoub, E. M., Harley, J. B., \& Cunningham, M. W. (1993). Poststreptococcal anti-myosin antibody idiotype associated with systemic lupus erythematosus and Sjögren's syndrome. Journal of Infectious Diseases, 168(4), 915-921.

3. Albishri, J. A. (2012). Chorea as a first manifestation in young patients with systemic lupus erythematosus who was initially diagnosed with rheumatic fever. Clinical Medicine Insights: Case Reports, 5, CCRep-S9143.

4. Diaz, C., Lim, M. A., Liu, C. A., Miwa, C. S., Tokunaga, D., Hamamura, F. D., ... \& Kurahara, D. (2018). Diagnosis of Systemic Lupus Erythematosus in a Polynesian Male with a History of Rheumatic Fever: A Case Report and Literature Review. Case reports in pediatrics, 2018.

Cite This Article: Jaouad Yousfi et al (2021). Rheumatic Fever and Systemic Lupus Erythematosus: A Rare Association. East African Scholars J Med Sci, 4(3), 76-77. 